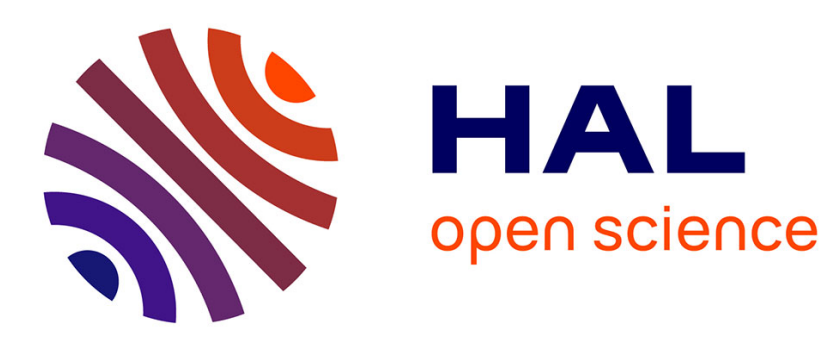

\title{
Downlink Capacity Gain Analysis of Mobile Relay in LTE-Advanced Network
}

\author{
Yangyang Chen, Xavier Lagrange
}

\section{To cite this version:}

Yangyang Chen, Xavier Lagrange. Downlink Capacity Gain Analysis of Mobile Relay in LTEAdvanced Network. CCNC 2014: 11th Annual IEEE Consumer Communications \& Networking Conference, Jan 2014, Las Vegas, United States. hal-00966743

\section{HAL Id: hal-00966743 https://hal.science/hal-00966743}

Submitted on 27 Mar 2014

HAL is a multi-disciplinary open access archive for the deposit and dissemination of scientific research documents, whether they are published or not. The documents may come from teaching and research institutions in France or abroad, or from public or private research centers.
L'archive ouverte pluridisciplinaire HAL, est destinée au dépôt et à la diffusion de documents scientifiques de niveau recherche, publiés ou non, émanant des établissements d'enseignement et de recherche français ou étrangers, des laboratoires publics ou privés. 


\section{Downlink Capacity Gain Analysis of Mobile Relay in LTE-Advanced Network}

\author{
Yangyang Chen \\ Institut Mines Telecom / Telecom Bretagne \\ IRISA \\ France \\ Email: yangyang.chen@telecom-bretagne.eu
}

\author{
Xavier Lagrange \\ Institut Mines Telecom / Telecom Bretagne \\ IRISA \\ France \\ xavier.lagrange@telecom-bretagne.eu
}

\begin{abstract}
In this paper, we analyze whether mobile relay could bring capacity gain to a LTE-Advanced network. The analysis is based on the Shannon formula modified for LTE. The Spatial Poisson Process is used to model the distribution of terminals and mobile relays in the cell. We consider two relay modes in the study: pure-relay mode and femto-like mode. Through the analysis we get the conclusion that penetration loss is a key factor to decide whether mobile relay can bring considerable capacity gain into the system. When penetration loss is $25 \mathrm{~dB}$, pure-relay mode can bring about $+30 \%$ capacity gain compared with traditional mode, femto-like mode can bring almost $+100 \%$ capacity gain compared with traditional mode.
\end{abstract}

Index Terms-Mobile Relay; Capacity Gain; Spatial Poisson Process; LTE;

\section{INTRODUCTION}

Relay has been considered as a key technology for 3GPP LTE-Advanced, as it can increase the network capacity, and provide coverage in new area [1]. There exist two types of relays, fixed relay and mobile relay [2]. Fixed relays are usually deployed at locations that are shrewdly chosen, such as shadow area or hot spot. They have already been standardized by 3 GPP in release 10 . Now in the context of ITS (Intelligent Transport System), the 3GPP is working on enhancing the relay functionality with mobile relays [3]. Mobile relays are mounted in a moving vehicle that provides wireless connectivity to users inside the vehicle via an indoor antenna. The backhaul connection to donor eNodeB is provided via an outdoor antenna.
One example use case is buses, as shown in Figure 1. The link between donor eNodeB and the relay is called the backhaul link, the link between the relay and the embedded UE is called the access link, and the link between the eNodeB and the UE is called the direct link. The buses are well shielded with coated windows, which leads to a rather high penetration loss between outdoor and in-vehicle. Furthermore, the vehicle can be anywhere in the cell without dedicated network planning like fixed relay. Therefore providing the required services to the massive users that are moving with the bus is much more challenging than typical mobile wireless environments.

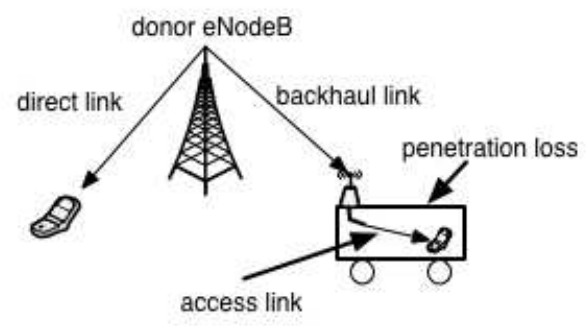

Fig. 1. Mobile relay mounted on a bus

Numerous studies have been done on performance evaluation of fixed relays. However, to the best of our knowledge very few studies have been done towards the scenario that deploying mobile relay in public transportation systems. In the previous 
related work about mobile relay [4][5][6]. [4] presented an simplified and ideal capacity analysis of fixed relay and mobile relay with SISO antenna configuration and without shadowing. [5] investigated the performance of dual-hop moving relay assisted transmission, but the scenario they considered was more related to point-to-point transmission, not a network. [6] investigated the data rate gain provided by one mobile relay deployed in a network with only one terminal, but how much capacity gain can be attained through mobile relays in a LTE network is still unknown.

Therefore in this paper, we focus on the capacity gain in a network deployed with mobile relays. The relay in this work is layer 3 relay specified by LTE [7]. The sections are organized as follows. Firstly, the system model is given in Section II, including some assumptions and analysis methods. Secondly, some analysis and calculations are given in Section III. Then the performance results are shown in Section IV. A conclusion is drawn in Section V.

\section{System Model}

\section{A. Spatial Model}

In this study we focus on a downlink LTE network with multi-user and multi-relay. We consider three types of nodes existing in the mobile relay scenario.

1) Mobile relays: which are mounted on public buses. Each relay has an attenuation outside the bus for the backhaul link, and an invehicle attenuation for the access link.

2) Ordinary UEs: which are outside the bus. They always connect to the eNodeB directly.

3) Embedded UEs: which are inside the bus.

When mobile relays are deployed, we assume all embedded UEs are connected to the LTE network through mobile relays. And we assume ordinary UEs and buses are uniformly distributed in the cell. The locations of mobile relays can be represented by the locations of buses. Embedded UEs are uniformly distributed inside the bus. But from the view of the cell, the UEs (ordinary and embedded) are not uniformly distributed in the cell. We use Spatial Poisson Process to model the distribution of mobile relays and UEs [8]. The related variables are listed in the Table 1.

Based on the Spatial Poisson Distribution, the probability to have $n_{u}$ UEs in area $A$ is:

$$
P\left(N_{U}=n_{u}\right)=\frac{\left(\rho_{U} A\right)^{n_{u}}}{n_{u} !} \mathrm{e}^{-\rho_{U} A} .
$$

The probability to have $n_{v}$ vehicles in area $A$ is:

$$
P\left(N_{V}=n_{v}\right)=\frac{\left(\rho_{V} A\right)^{n_{v}}}{n_{v} !} \mathrm{e}^{-\rho_{V} A} .
$$

\section{B. Interference Model}

In LTE network, the cell-edge terminals suffer from severe interferences from neighbor eNodeBs. Neighbor eNodeBs are the source of interferences. In 3GPP specified relay network, the relay utilizes the same frequency band for the access link and the backhaul link, so the access link and the backhaul link are separated in time domain. Due to this mechanism, two new types of interferences are brought in the mobile relay scenario. One is Relayto-Relay interference, the other one is Relay-to-UE interference, as shown in Figure 2.

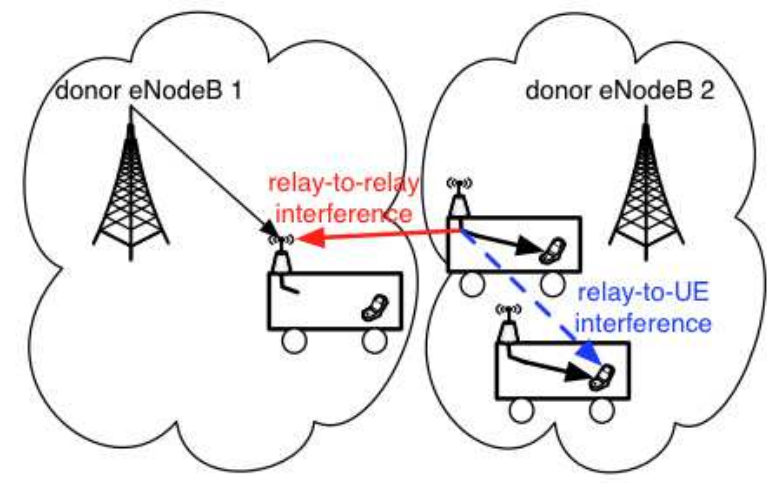

Fig. 2. Interferences in mobile relay scenario

1) Relay-to-Relay Interference: In FDD LTE, eNodeBs do not need to be synchronized in time domain [9]. All mobile relays under the same donor eNodeB are aligned, but mobile relays under different eNodeBs may be misaligned. For example, one mobile relay $A$ under eNodeB 1 is receiving data on the backhaul link, another mobile relay $B$ under 
TABLE I

THE TABLE OF THE VARIABLES FOR SPATIAL MODEL

\begin{tabular}{|c|c|}
\hline Variable & Define \\
\hline$A$ & The area of the cell $\left(\mathrm{km}^{2}\right)$ \\
\hline$N_{U}$ & the random variable stands for the number of UEs in the cell \\
\hline$n_{u}$ & the value of the random variable $N_{U}$ \\
\hline$N_{V}$ & the random variable stands for the number of buses in the cell \\
\hline$n_{v}$ & the value of the random variable $N_{V}$ \\
\hline$\rho_{V}$ & the density of buses in the cell \\
\hline$\rho_{U}$ & the density of UEs per $\mathrm{km}^{2}$ in the cell \\
\hline
\end{tabular}

eNodeB 2 is sending data on the access link, they are both in cell-edges and close to each other. In this case, mobile relay $A$ might suffer interference from mobile relay $B$. The Relay-to-Relay interference is shown as solid line in Figure 2. In our model, we assume all eNodeBs are synchronized, so this type of interference is not considered.

2) Relay-to-UE Interference: Another kind of interference is Relay-to-UE interference. When two mobile relays are transmitting to their embedded UEs, frequency reuse is applied on the access link. If they are close to each other, which is possible for two buses, the embedded UEs can be interfered by the neighbor mobile relay. It's shown as dotted line in Figure 2. This kind of interference is unique in mobile relay scenario. But since the transmitted power of mobile relay is relatively small, when two mobile relays are far away, this kind of interference is generally small. This interference is considered in our work.

\section{Propagation Model}

In mobile relay scenario the propagation loss can be divided into three parts: outdoor path loss, penetration loss $L_{\text {trans }}$ between outdoor and invehicle and in-vehicle path loss. Outdoor path loss is modeled by Okumura-Hata formula. Since the terminal inside the bus is close to the in-vehicle antenna of mobile relay. We consider free space propagation inside the bus. Path loss model is shown in Table II, $d$ (in $\mathrm{km}$ ) is the distance between two nodes.

The signal power at the receiver can be written with the general formula:

$$
P_{R}=P_{S} \frac{k 10^{\frac{\sigma_{s}}{10} \xi}}{d^{\alpha}}
$$

TABLE II

PROPAGATION LOSS

\begin{tabular}{|c|c|}
\hline outdoor path loss & $L_{\text {outdoor }}=141.78+35 \log _{10}(d)$ \\
\hline penetration loss & {$[10,30] \mathrm{dB}$} \\
\hline in-vehicle path loss & $L_{\text {in-vehicle }}=98.4+20 \log _{10}(d)$ \\
\hline
\end{tabular}

where $P_{S}$ is the transmitted power of the transmitter, $k$ and $\alpha$ are constant parameters deduced from propagation loss, $\xi$ is a standard normal random variable that models shadow fading and parameter $\sigma_{s}$ is the standard deviation of shadow fading.

\section{System Mode}

In this article we compare three modes: traditional mode, pure-relay mode and femto-like mode. The traditional mode is a baseline mode. There are no mobile relays, all terminals connect directly to eNodeB. In the pure-relay mode, when mobile relays transmit to embedded UEs in the access time slot, the eNodeB stops transmitting to ordinary UEs. But in the femto-like mode, frequency reuse is applied: in the access time slot eNodeB and relay transmit to ordinary UEs and embedded UEs respectively at the same time, as shown in Figure 3. So for embedded UEs in the femto-like mode the donor eNodeB is one source of the interferences.

\section{System ANALYSIS}

\section{A. The calculation of SINR}

In this section, we compute the SINR of the terminal.

1) SINR in the traditional mode: In this mode, the UE connects to the eNodeB directly. We consider outdoor path loss, penetration loss on the direct link, and the interferences from neighbor 


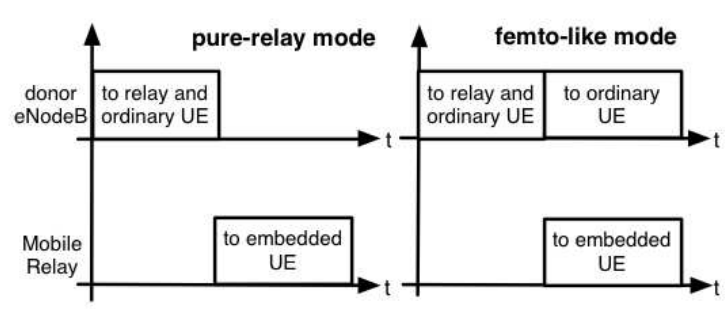

Fig. 3. The pure-relay mode vs The femto-like mode

eNodeBs. The SINR of UE $i$ in traditional mode is:

$$
\delta_{U E}^{i}=\frac{P_{S} k_{1} 10^{\frac{\sigma_{s 1}}{10} \xi}}{d^{\alpha_{1}}\left(\sum_{j} P_{j}+N\right)}
$$

where $P_{S}$ is the transmitted power of donor eNodeB, $P_{j}$ is the received power from other eNodeB $j, d$ is the distance between UE and donor eNodeB, $k_{1}=10^{-14.178} \times 10^{-L_{\text {trans }} / 10}, \alpha_{1}=3.5$ and $N$ is UE's thermal noise.

2) SINR in two relay modes: In relay network, the backhaul link and the access link are timedivision multiplexed, so the interferences are different in the backhaul time slot and in the access time slot. For ordinary UE, the SINR in the backhaul time slot remains the same as the traditional mode. For mobile relay, the signal that the mobile relay receives from donor eNodeB mainly suffers outdoor path loss. The SINR of mobile relay $k$ in the backhaul time slot is:

$$
\delta_{\text {relay }}^{k}=\frac{P_{S} k_{2} 10^{\frac{\sigma_{s 1}}{10} \xi}}{d^{\alpha_{1}}\left(\sum_{j} P_{j}+N_{\text {Relay }}\right)}
$$

where $P_{S}$ is the transmitted power of donor eNodeB, $P_{j}$ is the received power from neighbor eNodeB $j, d$ is the distance between relay and donor eNodeB, $k_{2}=10^{-14.178}, \alpha_{1}=3.5$ and $N_{\text {Relay }}$ is mobile relay's thermal noise.

In the access time slot, the signal that embedded UEs receives mainly suffers in-vehicle path loss. The interferences are from neighbor eNodeBs and other mobile relays. The SINR of embedded UE $i$ in the pure-relay mode is:

$$
\delta_{U E}^{i}=\frac{P_{\text {relay }} k_{3} 10^{\frac{\sigma_{s 2}}{10} \xi}}{d^{\alpha_{2}}\left(\sum_{j} P_{j}+\sum_{k} P_{\text {relay }, k}+N\right)}
$$

where $P_{\text {relay }}$ is the transmitted power of relay, $P_{j}$ is the received power from neighbor eNodeB $j$, $P_{\text {relay, } k}$ is the received power from neighbor relay $k, d$ is the distance between relay indoor antenna and embedded UE, $k_{3}=10^{-9.84}, \alpha_{2}=2$ and $N$ is UE's thermal noise. Note that, in femto-like mode donor eNodeB is one source of interferences for embedded UE. The received power from donor eNodeB for embedded UE $i$ should be added as interference in equation (6). For ordinary UEs in femto-like mode, in the access time slot relay is one source of interferences. For them the received power from relays should be added as interference in equation (4).

\section{B. Scheduling}

We consider equal bit rate scheduling, which means all UEs are served with the same data rate. We define the served data rate as the unified bit rate per second for each UE. The served date rate depends on the number of UEs and channel condition. The data rate on each link can reflect channel condition, which is calculated by Shannon formula modified for LTE and is limited by Modulation Coding Scheme (MCS) [10]. Note that in relay modes the data that mobile relay transmits to embedded UEs is, at most, the one that relay receives on the backhaul link. The related variables are listed in the Table 3 in the sequence of alphabet.

First we calculate the served data rate in traditional mode. The idea is to calculate how many bits can be transmitted per second for each UE. The inverse of the data rate can be seen as the time to transmit 1 bit. In traditional mode, eNodeB can serve the UEs all the time. So the served data rate $R_{\text {served }}$ is:

$$
R_{\text {served }}=\frac{1}{\sum_{i=1}^{N_{o}} \frac{1}{R_{o, i}}+\sum_{i=1}^{N_{b}} \sum_{j=1}^{N_{i, a}} \frac{1}{R_{e, j}}}
$$

Then in the pure-relay mode, eNodeB can only serve the UEs in the backhaul time slot, so $R_{\text {served }}$ 
TABLE III

VARIABLES FOR EQUAL BIT SCHEDULING

\begin{tabular}{|c|c|}
\hline Variable & Define \\
\hline \hline$N_{b}$ & the number of buses (mobile relays) in the cell \\
\hline$N_{i, a}$ & the number of UEs inside the bus associated with bus (mobile relay) $i$ \\
\hline$N_{o}$ & the number of ordinary UEs in the cell \\
\hline$R_{a, i}$ & the data rate for embedded UE $i$ on the access link \\
\hline$R_{b, i}$ & the data rate for mobile relay $i$ on the backhaul link \\
\hline$R_{e, j}$ & the data rate for UE $j$ inside the bus in traditional mode \\
\hline$R_{o, i}$ & the data rate for ordinary UE $i$ in traditional mode \\
\hline$R 1_{o, i}$ & the data rate for ordinary UE $i$ in the backhaul time slot \\
\hline$R 2_{o, i}$ & the data rate for ordinary UE $i$ in the access time slot \\
\hline$R_{\text {served }}$ & the served date rate for all UEs \\
\hline
\end{tabular}

is:

$$
R_{\text {served }}=\frac{1}{\sum_{i=1}^{N_{o}} \frac{1}{R 1_{o, i}}+\sum_{i=1}^{N_{b}} \sum_{j=1}^{N_{i, a}}\left(\frac{1}{R_{b, i}}+\frac{1}{R_{a, j}}\right)}
$$

In the femto-like mode, eNodeB can serve ordinary UEs all the time, so $R_{\text {served }}$ is:

$$
R_{\text {served }}=\frac{1}{\sum_{i=1}^{N_{o}} \frac{1}{R 1_{o, i}+R 2_{o, i}}+\sum_{i=1}^{N_{b}} \sum_{j=1}^{N_{i, a}}\left(\frac{1}{R_{b, i}}+\frac{1}{R_{a, j}}\right)}
$$

\section{Resource Allocation Model}

To simplify the resource allocation process, we allocate the resource in the manner of time, as is shown in Figure 4. In this model each mobile relay or UE take the whole system bandwidth, but because of different channel conditions, they consume different time resource.

First eNodeB allocates resources to mobile relays in the backhaul time slot. After that, eNodeB can keep allocating resources to ordinary UEs in the backhaul time slot. Then in the access time slot, mobile relays allocate resources to their embedded UEs. Also, in the femto-like mode donor eNodeB can allocate resources to unserved ordinary UEs simultaneously. The resource allocated to each node $i$ is calculated according to its channel condition:

$$
\alpha_{x, i}=\frac{R_{\text {served }}}{R_{x, i}}
$$

where $x=o 1, o 2, b, a$ stands for ordinary UE in the backhaul time slot, ordinary UE in the access time slot, mobile relay and embedded UE respectively.

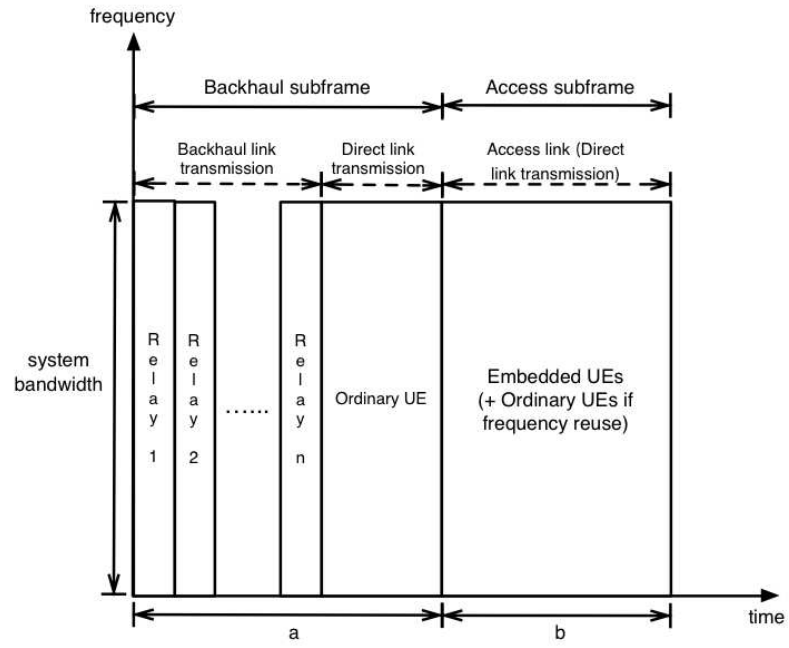

Fig. 4. Resource allocation in time domain

\section{Performance Results}

The performance of mobile relay is evaluated in this section. The evaluation is performed by Monte Carlo simulations. The total number of UEs and the number of vehicles (mobile relays) in the cell in each snapshot is a Poisson random parameter. Mobility and handover are not taken into account. Random shadowing is fixed in each snapshot.

The vehicles (per km of road) in France was 37 in 2010 [11]. This data include cars, buses, and freight vehicles. We only consider public buses in mobile relay scenario, the density of buses is set to 4 per $\mathrm{km}^{2}$. 
In the traditional mode, all UEs connect directly to the donor eNodeB. In the pure-relay mode and the femto-like mode the distance between the mobile relay and an embedded UE is assumed to be 20 meters (the bus is usually 40 meters long at most), and the transmitted power of mobile relay is set to $-10 \mathrm{dBm}$ [12]. The number of backhaul subframes in one radio frame is set to 6 . Some main parameters are listed in Table IV.

TABLE IV

SYSTEM PARAMETERS

\begin{tabular}{|c|c|}
\hline Parameter & Value \\
\hline \multicolumn{2}{|l|}{ Network parameters } \\
\hline Number of eNodeBs & 19 \\
\hline Radius from the center of the cell & $1 \mathrm{~km}$ \\
\hline The density of vehicles (per $\mathrm{km}^{2}$ ) & 4 \\
\hline The density of UEs $\left(\right.$ per $\left.\mathrm{km}^{2}\right)$ & 50 \\
\hline The number of UEs in a bus & 5 \\
\hline \multicolumn{2}{|l|}{ System parameters } \\
\hline Carrier Frequency & $2 \mathrm{GHz}$ \\
\hline System Bandwidth & $10 \mathrm{MHz}$ \\
\hline Bandwidth Efficiency $\cdot \eta$ & 0.65 \\
\hline SNR Efficiency & 0.95 \\
\hline Penetration loss & {$[10,30] \mathrm{dB}$} \\
\hline Outdoor Shadowing & $8 \mathrm{~dB}$ \\
\hline In-vehicle Shadowing & $4 \mathrm{~dB}$ \\
\hline \multicolumn{2}{|l|}{ eNodeB parameters } \\
\hline Transmit power & $46 \mathrm{dBm}$ \\
\hline Antenna Gain & $14 \mathrm{dBi}$ \\
\hline \multicolumn{2}{|c|}{ Mobile Relay parameters } \\
\hline Transmit power & $-10 \mathrm{dBm}$ \\
\hline Noise Figure & $5 \mathrm{~dB}$ \\
\hline \multicolumn{2}{|c|}{ User Equipment parameters } \\
\hline Noise Figure & $9 \mathrm{~dB}$ \\
\hline
\end{tabular}

\section{A. The Served Data Rate}

First of all, we compare the served data rate in three modes under different ratio of embedded UEs in the cell. The simulation result is shown in Figure 5 .

As shown in Figure 5, two relay modes have higher served data rate, this is because penetration loss is eliminated in relay modes. With the increase of the proportion of UEs inside the vehicle, more UEs suffer from penetration loss in the traditional mode, so the served data rate decreases. In the relay modes with the increase of UEs inside the vehicle, backhaul link increasingly becomes the bottleneck, so the served data rate also decreases.

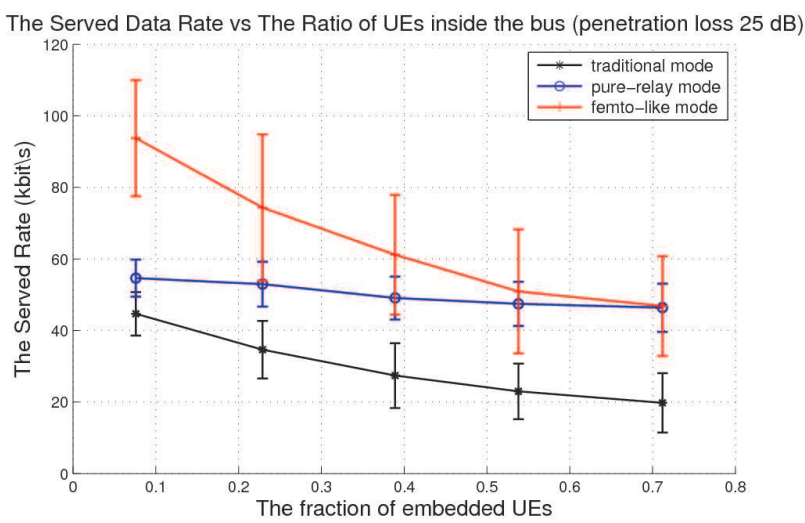

Fig. 5. the served data rate vs the fraction of embedded UEs (with $95 \%$ confidence interval)

\section{B. The Penetration Loss}

The Penetration loss affects the performance significantly. We compare system capacity in different modes under different penetration loss when the number of embedded UEs in a bus is fixed to 5 [13]. The System capacity is the sum of the achievable data rate of all served UEs. The results are shown in Figure 6.

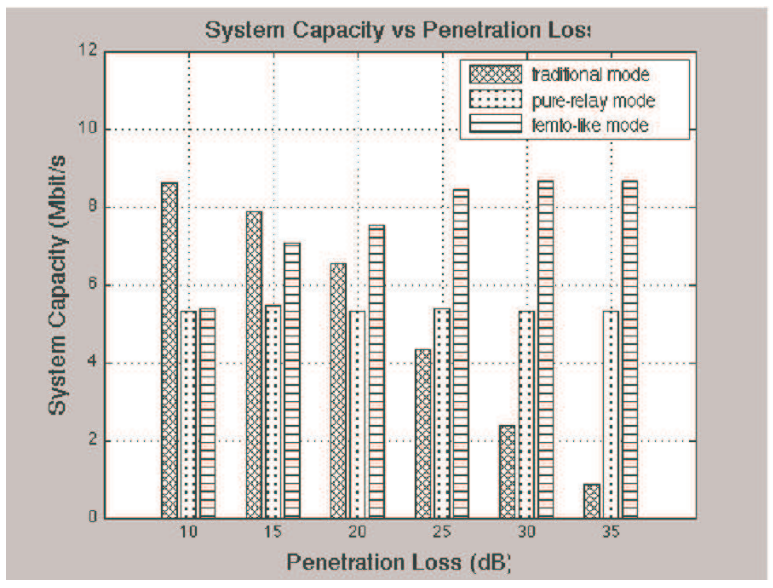

Fig. 6. System Capacity vs Penetration Loss

From the figure we can see, the larger penetration loss is, the higher capacity relay modes could achieve, then more capacity gain can be brought by mobile relays. This is because mobile 
relay eliminates the affect of penetration loss. In traditional mode, penetration loss deteriorates the signal. According to [9], penetration loss in vehicles is normally between 20 to $30 \mathrm{~dB}$. When penetration loss is $25 \mathrm{~dB}$, pure-relay mode can bring about $+30 \%$ capacity gain, femto-like mode can bring almost $+100 \%$ capacity gain compared with traditional mode, so capacity gain can be attained by deploying mobile relay in public transportation systems.

Also we can see femto-like mode can achieve higher system capacity than pure-relay mode. This is because frequency reuse is applied in femtolike mode, eNodeB and mobile relay can send data simultaneously, which improves the bandwidth efficiency.

\section{CONCLUSION}

In this paper, we investigate the capacity gain that mobile relay could bring into a public transportation system. The Spatial Poisson Process is used to model the distribution of terminals and buses (mobile relays) in the cell. We compare the performance of traditional mode, pure-relay mode and femto-like mode. The results show that penetration loss is a key factor to decide whether mobile relay could bring capacity gain into system. Normally higher system capacity can be achieved in the femto-like mode due to frequency reuse. When penetration loss is $25 \mathrm{~dB}$, pure-relay mode can bring about $+30 \%$ capacity gain compared with traditional mode, femtolike mode can bring almost $+100 \%$ capacity gain compared with traditional mode. Furthermore, in our study we assume mobile relay has the same receiver as the UE, which means the performance of mobile relay can be improved by using better receivers.

\section{ACKNOWLEDGMENT}

This work was performed within project SYSTUF, which is subsidized by the french ministry of Industry in the framework of the AMI ITS program.

\section{REFERENCES}

[1] 3GPP TR36.814, "Further advancements for E-UTRA physical layer aspects," v9.0.0, Mar. 2010.
[2] Cheng-Xiang Wang; Xuemin Hong; Xiaohu Ge; Xiang Cheng; Gong Zhang; Thompson, J.; , "Cooperative MIMO channel models: A survey," Communications Magazine, IEEE, vol.48, no.2, pp.80-87, February 2010.

[3] 3GPP: Overview of 3GPP Release 12 V0.0.5, Sep. 2012.

[4] Huang Lin; Daqing Gu; Wenbo Wang; Hongwen Yang; , "Capacity analysis of dedicated fixed and mobile relay in LTE-Advanced cellular networks," Communications Technology and Applications, 2009. ICCTA '09. IEEE International Conference on, pp.354-359, 16-18 Oct. 2009.

[5] Sui, Y.; Papadogiannis, A.; Svensson, T., "The Potential of Moving Relays - A Performance Analysis," Vehicular Technology Conference (VTC Spring), 2012 IEEE 75th , vol., no., pp.1,5, 6-9 May 2012.

[6] Yangyang Chen; Lagrange Xavier, "Downlink data rate gain provided by a mobile relay for LTE-advanced," Wireless Personal Multimedia Communications (WPMC), 2013 16th International Symposium on , vol., no., pp.1,5, 24-27 June 2013.

[7] M. Iwamura, H. Takahashi, and S. Nagata, Relay technology in LTE- Advanced, NTT DoCoMo Technical J., vol. 12, no. 2, pp. 2936, 2009.

[8] Andrews, J.G.; Ganti, R.K.; Haenggi, M.; Jindal, N.; Weber, S., "A primer on spatial modeling and analysis in wireless networks," Communications Magazine, IEEE , vol.48, no.11, pp.156,163, November 2010.

[9] H. Holma and A. Toskala, LTE for UMTS - Evolution to LTE-Advanced, Wiley, 2011.

[10] Mogensen, P.; Wei Na; Kovacs, I.Z.; Frederiksen, F.; Pokhariyal, A.; Pedersen, K.I.; Kolding, T.; Hugl, K.; Kuusela, M., "LTE Capacity Compared to the Shannon Bound," Vehicular Technology Conference, 2007. VTC2007-Spring. IEEE 65th , vol., no., pp.1234,1238, 22-25 April 2007.

[11] The World Bank, "Vehicles (per km of road)," [online], http://data.worldbank.org/indicator/IS.VEH.ROAD.K1 (Accessed: 23 Jan 2013).

[12] Claussen, H.; Ho, L.T.W.; Samuel, L.G.; , "Selfoptimization of coverage for femtocell deployments," Wireless Telecommunications Symposium, 2008. WTS 2008, pp.278-285, 24-26 April 2008.

[13] Yutao Sui; Vihriala, J.; Papadogiannis, A.; Sternad, M.; Wei Yang; Svensson, T., "Moving cells: a promising solution to boost performance for vehicular users," Communications Magazine, IEEE , vol.51, no.6, pp., June 2013. 